\title{
RESPIRATORY QUOTIENTS OF BOVINE SPERMATOZOA
}

\author{
G. W. SALISBURY AND J. R. LODGE \\ Department of Dairy Science, University of Illinois, Urbana, U.S.A.
}

(Received 7th September 1962)

\begin{abstract}
Summary. The oxygen consumption of sixteen samples of ejaculated bovine spermatozoa in diluted semen was more rapid in the presence of the respired carbon dioxide than it was for similar spermatozoa respiring in the presence of alkali to absorb the evolved carbon dioxide. Thus, respiratory quotients for seminal spermatozoa calculated from oxygen consumptions obtained by the direct method of Warburg, in the absence of respired carbon dioxide, are in error, being larger than is characteristic of the substrate oxidized.

The oxygen consumption of seventeen samples of ejaculated bovine spermatozoa washed free from seminal plasma was not influenced to the same degree by the absence of the respired carbon dioxide. However, the influence was sufficient to question the accuracy of the direct method of Warburg for measuring respiratory quotients for bovine spermatozoa.
\end{abstract}

\section{INTRODUCTION}

Since Humphrey \& Mann (1949) found that ram spermatozoa consumed more oxygen from a gaseous mixture containing $5 \%$ carbon dioxide than from either air or $100 \%$ oxygen, and Salisbury \& Kinney (1957) observed a marked stimulation to aerobic glycolysis by bull spermatozoa in the presence of respired carbon dioxide, the usual technique for determination of the respiratory quotients of spermatozoa has been suspect. In the direct method of Warburg (Umbreit, Burris \& Stauffer, 1957), commonly used for this determination, the oxygen consumption of the tissue is measured in the continual presence of an alkali which absorbs carbon dioxide as it is produced; whereas the carbon dioxide output of the respiring tissue is, of course, measured in another flask in which the concentration of the evolved carbon dioxide is continuously increasing. If this evolved carbon dioxide is, in fact, not only stimulatory to the aerobic glycolysis by bovine spermatozoa, but stimulatory to respiration as well, the oxygen uptake measured in the presence of alkali, and thus in the absence of respired carbon dioxide, would be less than that actually being consumed by the tissue in the flask providing evidence on carbon dioxide output, increasing the carbon dioxide: oxygen ratio. The net effect of this disparity would be respiratory quotients which are larger than is typical of the substrate being oxidized. 
The use of Dixon-Keilin flasks (Dixon, 1952) allowed respiration to proceed in the presence of the respired carbon dioxide and the later introduction of alkali at the desired time permitted volumetric measurements of both the oxygen and carbon dioxide content of the flask. Thus calculations of the gaseous exchange by the cells were possible.

This report presents the results of experiments designed to test the comparative validity of respiratory quotients determined by the direct method of Warburg and by the method of Dixon for bovine spermatozoa in semen and washed free of seminal plasma.

\section{METHODS AND MATERIALS}

The methods used for semen collection, determination of sperm concentration, and sperm motility have been reported (Bishop \& Salisbury, 1955). The measurement of oxygen uptake and carbon dioxide produced in the same flask was by the method of Dixon (1952). Single side-arm Dixon-Keilin flasks of about 17-ml capacity were used with Warburg manometers. One flask was used for each of the following: the initial carbon dioxide content which included the bound carbon dioxide and the carbon dioxide in the gas phase; the carbon dioxide produced and the oxygen uptake for 1-, 2-, 3- and 4-hr periods in the presence of carbon dioxide; and one flask for measurement of oxygen uptake in the absence of carbon dioxide throughout the incubation. Two flasks were used as thermobarometers. The flasks were incubated at $37^{\circ} \mathrm{G}$. The main compartment of the flasks contained $1 \mathrm{ml}$ of the diluent (saline solution or saline solution with $0.5 \%$ fructose) and $0.2 \mathrm{ml}$ of semen or washed cell suspension, containing about $3 \times 10^{8}$ sperm cells, which was added just prior to placing the flasks on the manometers. The side-arm contained $0.2 \mathrm{ml}$ of $3 \mathrm{~N}-\mathrm{H}_{2} \mathrm{SO}_{4}$ for use in releasing the bound carbon dioxide and to stop metabolism at the end of each time-period. Twenty per cent potassium hydroxide was used to absorb the carbon dioxide and air was the gas phase.

The final carbon dioxide values were corrected for the initial carbon dioxide content to give the actual amount of carbon dioxide produced during the given time-period. The oxygen uptake values were corrected for the initial gas phase carbon dioxide content which would be absorbed by potassium hydroxide after its introduction and otherwise would be measured as a volume equivalent of oxygen. The corrected carbon dioxide and oxygen values were converted to microlitres per $10^{8}$ cells and the respiratory quotients (R.Q.) were calculated from these volumes. The statistical techniques employed were those described by Snedecor (1956).

The experiment reported here was conducted with ejaculated spermatozoa in semen (sixteen observations) diluted with $0.9 \%$ saline solution and with saline solution containing $0.5 \%$ fructose or with twice-washed ejaculated spermatozoa (seventeen observations) resuspended in the same two media. An argument advanced by MacLeod (1941), relating to human semen, that reliable basic evidence for calculation of R.Q. could not be obtained for spermatozoa in semen because it contains bicarbonate and other buffers, was overcome in the experiment reported here by tipping excess acid, sufficient to 

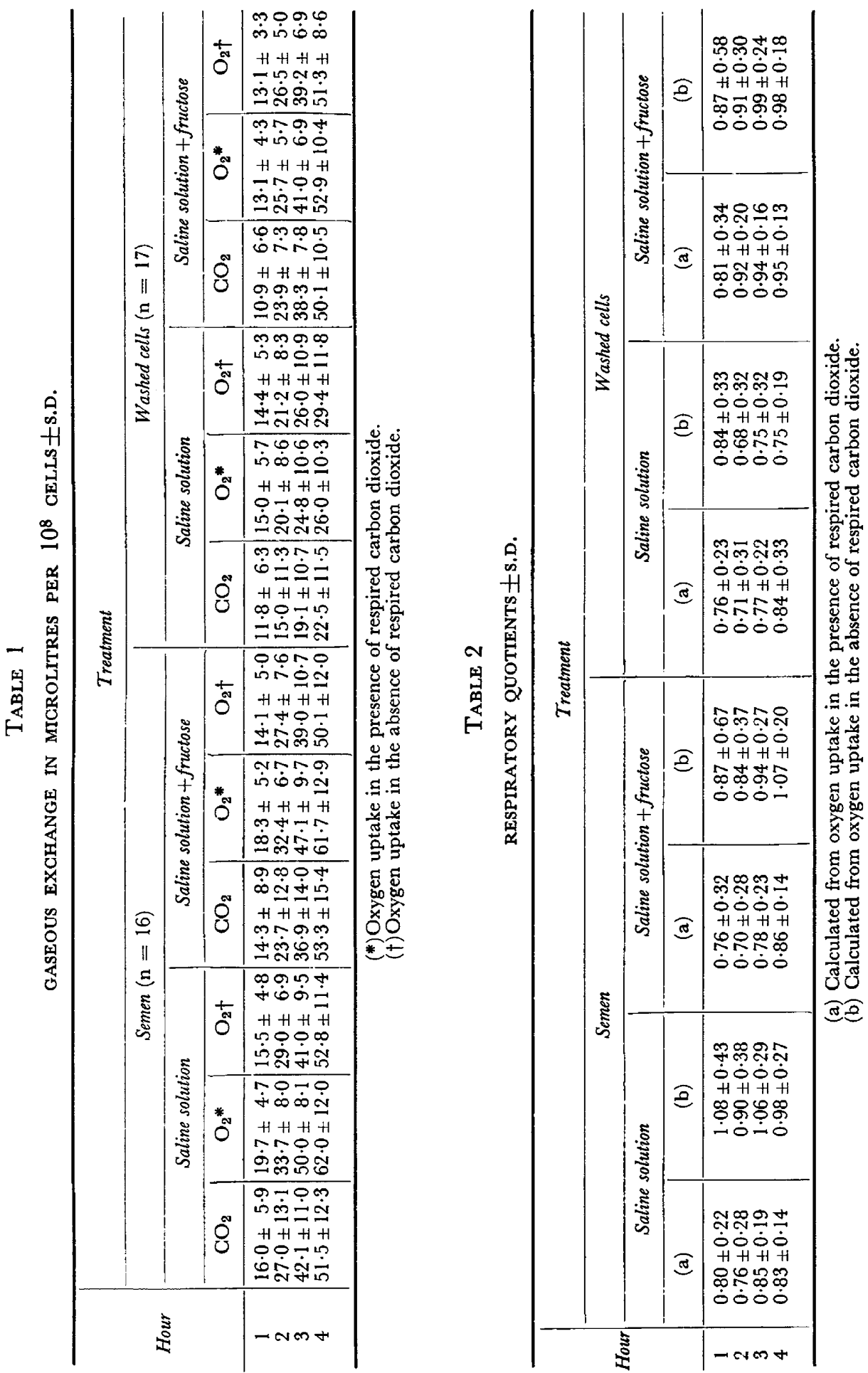
evolve all bound carbon dioxide, from the side-arm of the initial flask and each of the other flasks at the termination of each metabolic experiment.

\section{RESULTS}

The experiment was designed to test the reliability of the direct method of Warburg (Umbreit et al., 1957) against the method of Dixon (1952) for measurements of gaseous exchange and determinations of R.Q. with and without added fructose. The mean gaseous exchange for these four treatments at the end of each of the $4 \mathrm{hr}$ and their standard deviations are shown in Table 1. The coefficients of variability for the gas exchange at the end of the 4-hr period are shown in Table 3. Table 2 gives the R.Q. values calculated for each of the methods of measuring oxygen consumption.

TABLE 3

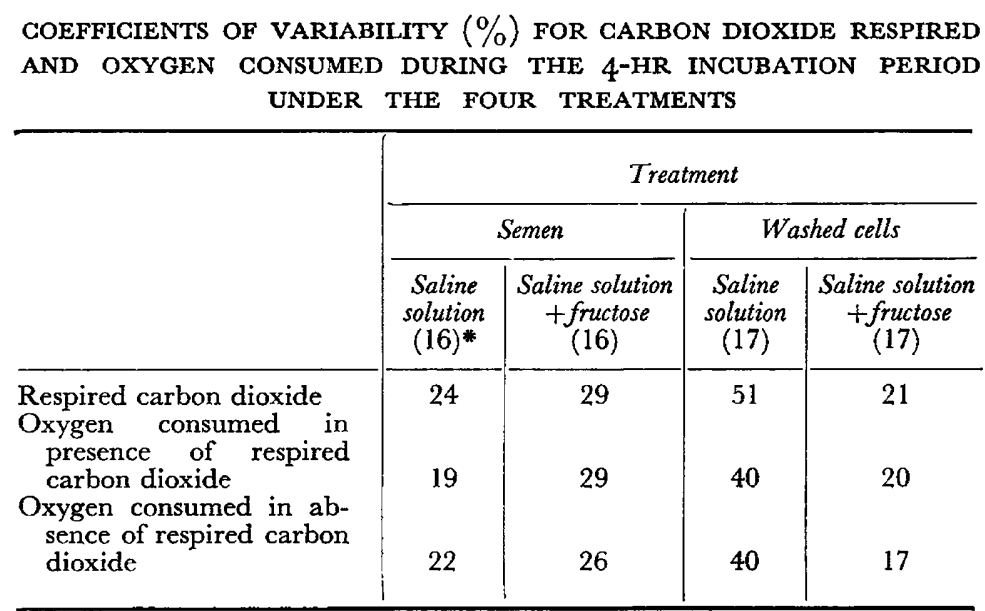

* Number of observations in parentheses.

The coefficients of variability for gas exchange fall into two general classes. One group represents variations in gaseous exchange of cells respiring in the presence of seminal plasma or of fructose, while the other, being the larger of the two classes of values, represents variations which occur in the oxidation of endogenous energy reserves (washed cells diluted with saline solution). The greater variability of gaseous exchange during endogenous respiration (in the absence of extracellular substrate) most likely is due to the variable and uncontrolled period of contact between the cells and the seminal plasma before centrifugation and washing and to the inherent differences among semen samples in intracellular substrate from the several bulls involved.

The coefficients of variability shown in Table 3 for the sum of the exchange at the end of the 4-hr-incubation period do not show the effect of time on the variability among samples from one to another treatment. However, the standard deviations shown in Table 2 do show the effect of time. The coefficients of variability for all measures of gaseous exchange decrease from the end of the first to the end of the 4th hour for all treatments containing exogenous 
substrate, but no such decreasing trend is shown for the variability of gaseous exchange of the sperm cells oxidizing endogenous reserves. The variability for these measures was of the same general magnitude at the end of each of the periods of incubation. Though the coefficients of variability for the oxygen consumption were similar whether or not the respiration occurred in the presence or in the absence of the respired carbon dioxide, this was not true for the absolute oxygen consumption. At the end of the 4-hr-incubation period in the diluted semen, the oxygen consumption per $10^{8}$ spermatozoa (Table 1) in the presence of the respired carbon dioxide was $17 \%$ greater in saline solution and $23 \%$ greater in saline solution plus fructose than it was in these two diluents in the absence of respired carbon dioxide. These differences are highly significant statistically, and the effect of these differences on calculated respiratory quotients is shown in Table 2.

The situation with washed cells is somewhat different, there being no marked differences in oxygen consumption in the presence or absence of respired carbon dioxide either in saline solution alone or when fructose was provided as the substrate. In fact, for any of the time-periods shown in Table 1, the only differences for oxygen consumption by washed cells which are statistically significant at any of the time-periods are those differences between endogenous respiration and respiration in the presence of fructose after the 1st hour. The differences shown for these two treatments at the end of the 4-hr period are highly significant $(P \leqslant 0.001)$ for respired carbon dioxide and for oxygen consumption both in the presence and absence of respired carbon dioxide. However, for the washed spermatozoa oxidizing endogenous reserves (in saline solution without added fructose), oxygen uptake at the end of the 1st hour in the absence of respired carbon dioxide is less than that occurring in its presence. At the end of the 4-hr period the reverse is true. This observation suggests that minimal amounts of carbon dioxide might be necessary to initiate optimum respiration in washed bovine spermatozoa as well as stimulating the metabolism of those cells in the presence of seminal plasma. This is to be the subject of future investigations. Also, it suggests that the rate of oxygen uptake as reflected in the constants for the slope of the regression of oxygen uptake on time might be statistically different under the two experimental treatments of presence and absence of respired carbon dioxide with or without added carbohydrate substrate.

In fact, if the gaseous exchange with time showed no significant departure from linearity, and this would depend in part on the vigour of the spermatozoan samples involved, a precise method of calculation of R.Q. would be that of regressing each gas exchange on time and comparing their appropriate ratio at specific time intervals. The advantage of such a technique is that all observations are used to estimate the slope of the regression line and the impact of observations widely deviating from the means are dampened. This has been done for the regression lines for washed cells shown in Text-fig. 1 and for the calculation of all R.Q. shown in Table 4. Statistical tests show no significant deviation from linearity with time for any respiratory measurement in these experiments, but this would not necessarily be the case under other conditions for other semen samples. 
Appropriate tests of the statistical significance of the regression constants expressing the slopes of the oxygen uptake showed a highly significant $(P \leqslant$ $0.001)$ difference for that occurring in the presence of respired carbon dioxide

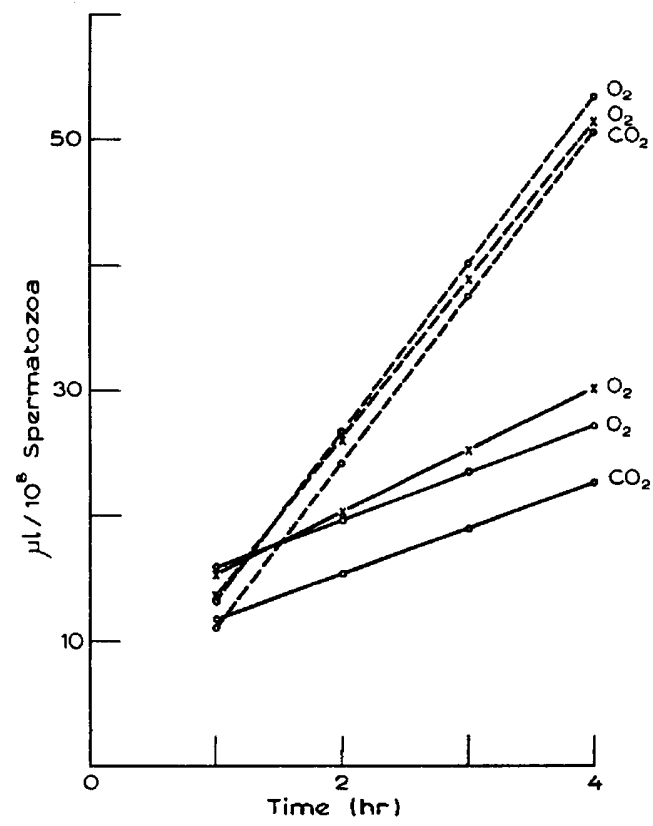

TEXT-FIG. 1. Linear regression of oxygen uptake and carbon dioxide production of washed spermatozoa.

$-=$ without fructose; $-\cdots-.-=$ with fructose;

$0=$ with carbon dioxide; $x=$ without carbon dioxide.

\section{TABLE 4}

RESPIRATORY QUOTIENTS GALGULATED FROM LINEAR REGRESSION

\begin{tabular}{|c|c|c|c|c|}
\hline \multirow{2}{*}{ Treatment } & \multicolumn{4}{|c|}{ Hour } \\
\hline & 1 & 2 & 3 & 4 \\
\hline $\begin{aligned} \text { Washed cells }+ \text { saline solution }(+) & (+)\end{aligned}$ & $\begin{array}{l}0.74 \\
0.76\end{array}$ & $\begin{array}{l}0.78 \\
0.75\end{array}$ & $\begin{array}{l}0.77 \\
0.75\end{array}$ & $\begin{array}{l}0.83 \\
0.75\end{array}$ \\
\hline $\begin{array}{l}\text { Washed cells }+ \text { saline solution }(+) \\
\quad+\text { fructose }\end{array}$ & $\begin{array}{l}0.85 \\
0.81\end{array}$ & $\begin{array}{l}0.91 \\
0.92\end{array}$ & $\begin{array}{l}0.94 \\
0.96\end{array}$ & $\begin{array}{l}0.95 \\
0.98\end{array}$ \\
\hline Semen + saline solution & $\begin{array}{l}0.81 \\
1.01\end{array}$ & $\begin{array}{l}0 \cdot 82 \\
0.99\end{array}$ & $\begin{array}{l}0.83 \\
0.99\end{array}$ & $\begin{array}{l}0.83 \\
0.98\end{array}$ \\
\hline $\begin{array}{l}\text { Semen + saline solution }+ \\
\text { fructose }\end{array}$ & $\begin{array}{l}0.69 \\
0.84\end{array}$ & $\begin{array}{l}0.78 \\
0.96\end{array}$ & $\begin{array}{l}0.82 \\
1.00\end{array}$ & $\begin{array}{l}0.84 \\
1.02\end{array}$ \\
\hline
\end{tabular}

(+) Calculated from oxygen uptake in presence of carbon dioxide.

(-) Calculated from oxygen uptake in absence of carbon dioxide.

as compared to that in its absence for washed spermatozoa, both in the absence of and in the presence of exogenous substrate. Similarly, the slope of the regression line of oxygen uptake in the absence of carbon dioxide with time was 
significantly different from the slope of the regression line of respired carbon dioxide on time, but the slope of the regression line of oxygen uptake on time in the presence of the respired carbon dioxide and that of the respired carbon dioxide with time were not significantly different.

The next evidence obtained for an estimate of the reliability of R.Q. calculated from such data was to determine whether or not the carbon dioxide respired and oxygen consumed, although highly variable among samples, was highly correlated. Calculations for the 4-hr total gaseous exchange indicated that when the oxygen figures were those obtained in the presence of carbon dioxide, the two are correlated least for the cells in semen to which only saline solution was added, $r=0.81$, and most for the endogenous respiration of washed cells suspended in saline solution, $r=0.95$, the coefficients for the two treatments in which fructose was added were intermediate (semen, $r=0.88$; washed cells, $r=0.84$ ) between these two extremes. All of these coefficients are highly significantly different from zero.

\section{DISCUSSION}

From these experiments it is apparent that the direct method of Warburg cannot be used for accurate measurement of the respiratory quotients of respiring bovine spermatozoa. In the presence of seminal plasma, such respiratory quotients are biased and too large for the oxygen uptake is uniformly depressed throughout the 4-hr-incubation period by the absence of the respired carbon dioxide. For washed spermatozoa with or without an added substrate, the direction of the bias introduced by the technique is a function of time, being towards a larger R.Q. than the true value in the initial stages of the incubation but towards a lower value, especially for endogenous respiration, as the incubation proceeds.

The method of Dixon (1952) in which oxygen uptake takes place in the presence of the respired carbon dioxide, gives precise results free from the routine bias and the directly observable errors of the direct method of Warburg.

As noted above and as can be seen in Text-fig. 1, the parallel lines for regression of respired carbon dioxide and oxygen uptake in the presence of the respired carbon dioxide on time, lead to one inevitable consequence-R.Q. values which increase in magnitude with time.

The authors at present are inclined to explain the increase in R.Q. with time of incubation on the premise that an initial amount of carbon dioxide is removed from the gaseous exchange by the spermatozoa for metabolic purposes. With continued incubation and establishment of an equilibrium between the intracellular and extracellular carbon dioxide content, the amount of oxygen consumed and carbon dioxide respired becomes constant. Thus the absolute difference in the oxygen consumed and the carbon dioxide respired for a given time-period after the 1st hour, remains constant, but the percentage this constant difference is of the accumulated gaseous exchange decreases, resulting in increased respiratory quotients. That carbon dioxide is actually consumed by spermatozoa is supported by observations showing less carbon dioxide present in flasks after a short period of incubation for some samples 
(Lodge \& Salisbury, 1963) and more recently the demonstration of the need for carbon dioxide in anaerobic glycolysis of sperm cells (Lodge \& Salisbury, 1962). If a portion of the respired carbon dioxide is consumed by the spermatozoa, then the R.Q. as an estimate of the nature of the substrate being oxidized by spermatozoa can be only a rough approximation to the true value.

\section{AGKNOWLEDGMENT}

This research was supported in part by a grant from the Rockefeller Foundation.

\section{REFERENCES}

Bishop, M. W. H. \& Salisbury, G. W. (1955) Effect of dilution with saline and phosphate solutions on oxygen uptake of bull semen. Amer. 7. Physiol. 181, 114.

Drxon, M. (1952) Manometric methods, 3rd edn. Cambridge University Press.

Humphrey, G. F. \& MANN, T. (1949) Studies on the metabolism of semen. 5. Citric acid in semen. Biochem. 7. 44, 97.

LODGE, J. R. \& SAlrSBURY, G. W. (1962) Initiation of anaerobic metabolism of mammalian spermatozoa by carbon dioxide. Nature, Lond. 195, 293.

Lodge, J. R. \& Salisbury, G. W. (1963) Factors influencing metabolic activity of bull spermatozoa. VI. Metabolic $\mathrm{CO}_{2}$ and fructose. F. Dairy Sci. (In press).

MacLeod, J. (1941) The metabolism of human spermatozoa. Amer. J. Physiol. 132, 193.

Salisbury, G. W. \& KinNey, W. C. JR. (1957) Factors influencing metabolic activity of bull spermatozoa. III. pH. 7. Dairy Sci. 40, 1343.

SNEDEcor, G. W. (1956) Statistical methods, 5th edn. Iowa State College Press, Ames.

Umbrett, W., Burris, R. H. \& Stauffer, J. F. (1957) Manometric techniques, 3rd edn. Burgess, Minneapolis. 